\title{
Immunosensor for the Diagnostics of Autoimmune Hemolytic Anemia (AlHA) Based on Immobilization of a Monoclonal Antibody on a Layer of Silk Fibroin
}

\author{
Marli L. Moraes ${ }^{1}$, , Lais R. Lima², Josy C. Vicentini-Oliveira ${ }^{3}$, Aparecida Vitória G. de Souza ${ }^{3}$, \\ Osvaldo N. Oliveira Jr. ${ }^{4}$, Elenice Deffune ${ }^{3}$, and Sidney J. L. Ribeiro ${ }^{2}$ \\ ${ }^{1}$ Universidade Federal de São Paulo, Instituto de Ciência e Tecnologia, São José dos Campos, SP 12231-280, Brazil \\ ${ }^{2}$ Universidade Estadual Paulista, Instituto de Química, Araraquara, SP 14800-060, Brazil \\ ${ }^{3}$ Universidade Estadual Paulista, Hemocentro de Botucatu, Botucatu, SP 18618687, Brazil \\ ${ }^{4}$ Universidade de São Paulo, Instituto de Física de São Carlos, São Carlos, SP 13566-590, Brazil
}

\begin{abstract}
The diagnostics of the autoimmune hemolytic anemia (AIHA), a rare disease caused by autoantibody-induced hemolysis, is still prone to false positives for it is based on visual observation in the so-called Direct Coombs test. In this study, we developed a specific IgG hemolysis immunosensor produced with layer-by-layer (LbL) films containing a monoclonal antibody against human immunoglobulin (mAbIMUG) deposited along with a layer of silk fibroin (SF) derived from Bombyx mori cocoons. Adsorption of mAbIMUG on a SF layer was confirmed by the fluorescence emission band at $326 \mathrm{~nm}$. Immunosensors were prepared with LbL films deposited on interdigitated gold electrodes for impedance spectroscopy and on screen printed carbon electrodes for electrochemical measurements. When the SF/mAbIMUGLbL film was exposed to healthy red blood cells (RBCs), no cell binding was observed by the optical microscopy images. In addition, no major changes were observed in the signals of the square wave voltammogram and in the impedance spectra. In contrast, the electrochemical signal was significantly increased and the dielectric loss curve shifted for the sensing units containing RBCs with the antibody attached on the surface ("sick cells"). Furthermore, cell attachment was so strong that optical images still showed covered electrodes even after washing in PBS buffer. The detection with two distinct methods seems promising for an effective diagnosis of AlHA.
\end{abstract}

Keywords: Immunosensor, Layer-By-Layer Films, Monoclonal Antibody, Autoimmune Hemolytic Anemia.

\section{INTRODUCTION}

Extravascular hemolysis can occur in three clinical situations, namely autoimmune hemolytic anemia, hemolytic transfusion reactions (HTR) and immune-mediated fetal maternal allo-sensitization. ${ }^{1}$ The therapeutic choice for these problems requires fast, accurate diagnostics, which today is performed with the direct Coombs test or direct antiglobulin test (DAT). 2,3 Autoimmune hemolytic anemia (AIHA) is a rare disease caused by autoantibody-induced hemolysis leading to premature destruction of circulating red blood cells (RBCs) and consequently to insufficient plasma concentration. ${ }^{4-7}$ AIHA was the first autoimmune

\footnotetext{
*Author to whom correspondence should be addressed.
}

disorder for which an autoantibody was clearly shown to be involved in its pathogenesis. Hemolysis can occur by AIHA, HTR and hemolytic disease of the newborn (HDN). HTR and HDN are caused by allo-antibodies, i.e., antibodies are directed against high-incidence antigens, acting on allogenic RBCs. ${ }^{5,8}$ They are the consequence of transfusion of incompatible blood and passage of maternal antibodies through the placental barrier, destroying antigens expressed on the erythrocyte membrane of the fetus. ${ }^{7}$

The diagnostics of AIHA includes a combination of clinical symptoms attributable to anemia, such as fatigue, weakness, and pallor, and laboratory tests to detect RBC hemolysis. ${ }^{1,5,6}$ The first antiglobulin test for detecting antibodies in the red cell surface was developed by 
Coombs et al. ${ }^{9}$ and since then techniques for AIHA diagnosis are based on agglutination between RBC antigen and antibody. ${ }^{6}$ There are two methods of the Coombs test, the direct Coombs test, also known as direct antiglobulin test (DAT), and the indirect Coombs test. One is used for autoimmune hemolytic anemia and the other for hemolytic transfusion reaction and hemolytic disease of the newborn. ${ }^{1,4}$ Coombs test is prone to false positives or false negatives since the results may be affected by external factors such as temperature, sample preparation, incubation time, in addition to involving visual observation of an operator. Immunosensors can be a viable alternative to improve AIHA detection, since the transduction mechanism may be optical and/or electrical.

Immunosensors have been explored in clinical diagnostics owing to their specificity and sensitivity, being based on detecting the binding of complementary antigens and antibodies. ${ }^{11-15}$ In this study, we investigated a specific AIHA immunosensor produced with layer-by-layer (LbL) films ${ }^{16}$ containing a monoclonal antibody against human immunoglobulin (mAbIMUG) and silk fibroin (SF) derived from Bombyx mori cocoons. SF was chosen because it has been proven a suitable matrix for immobilizing biomolecules in LbL films, ${ }^{17-19}$ while a monoclonal antibody offers greater specificity and reproducibility than polyclonal antibodies. ${ }^{20}$ Two principles of detection were employed here: impedance spectroscopy and square wave voltammetry measurements.

\section{MATERIALS AND METHODS}

\subsection{Materials}

Silk fibroin (SF) was extracted from the cocoons of the Bombyx mori silkworm silk supplied by Bratac S.A., Brazil, using the procedures described by Rockwood et al. ${ }^{21}$ The cocoons were boiled during $30 \mathrm{~min}$ in $0.02 \mathrm{~mol} \cdot \mathrm{L}^{-1} \mathrm{Na}_{2} \mathrm{CO}_{3}$ solution at a concentration of $5 \mathrm{~g} \cdot \mathrm{L}^{-1}$, and washed with Milli-Q water to remove sericin. SF was then dissolved in $\mathrm{CaCl}_{2} / \mathrm{CH}_{3} \mathrm{CH}_{2} \mathrm{OH} / \mathrm{H}_{2} \mathrm{O}$ $(1: 2: 8, \mathrm{~m} / \mathrm{m} / \mathrm{m})$ solution at $60{ }^{\circ} \mathrm{C}$, and dialyzed with Milli-Q water using a cellulose acetate membrane at room temperature for $48 \mathrm{~h}$. For the SF film preparation an aqueous solution at concentration of $0.2 \%(\mathrm{~m} / \mathrm{m})$ was used.

The monoclonal antibody against human immunoglobulin (mAbIMUG) was produced at the Cellular Engineering Laboratory (UNESP/Botucatu) after immunization of five $\mathrm{BALB} / \mathrm{c}$ female mice with the suspension containing purified human $\operatorname{IgG}$ and aluminum hydroxide adjuvant on days $0,7,14$ and 21; on day 28 they received a booster without adjuvant. The mice were euthanized, and the spleen cells were collected and fused with NS1 myeloma cells obtained by Köhler and Milstein ${ }^{22}$ from the Laboratoire de Genie D' Institute National de Transfusion Sanguine (INTS), Agence Française du Sang, Paris. The fused cells in culture medium from mAb production (RPMI medium/HAT supplement $/ 20 \%$ Fetal Bovine Serum-FBS, Gibco $\left.{ }^{\circledR}\right)$ were dispensed to 96-well tissue culture plates and incubated at $37{ }^{\circ} \mathrm{C}$ in a humidified environment with $5 \% \mathrm{CO}_{2}$. Supernatants from hybridomas that grew up were collected and assayed by Indirect Antiglobulin Test (IAT). Briefly, for the sensitization of erythrocytes with human IgG antibody, blood samples from healthy donors with erythrocyte phenotyping cceedd (negative $\mathrm{RhD}$ phenotype/rr) were incubated with serum from patients with high titers $(>1 / 128)$ of anti-c, IgG class. After $30 \mathrm{~min}$ of incubation at $37{ }^{\circ} \mathrm{C}$, the samples were washed three times in isotonic saline solution (ISS) and resuspended in the same buffer (5\%). Culture supernatants $(\mathrm{mAb})$ were tested in the $2: 1(\mathrm{v} / \mathrm{v})$ ratio with the sensitized samples.

The hybridomas yielding a positive response in IAT were cloned by limiting dilution, ${ }^{23}$ and supernatants from wells showing single cell colonies were re-assayed by IAT. The clones were grown in culture medium for $\mathrm{mAb}$ production and frozen at $-196{ }^{\circ} \mathrm{C}$ in FBS with $10 \%$ dimethyl sulfoxide (DMSO). The expanded clones were injected into the peritoneal cavity of 2 month old BALB/c mice that have been primed with $1 \mathrm{~mL}$ Pristane (Sigma $\left.{ }^{\circledR} \mathrm{P} 2870\right)$ 15 days earlier. Ascitic fluid was taken and purified by affinity chromatography (AKTA Prime, GE Healthcare ${ }^{\circledR}$ ).

For RBCs preparation, human red blood cells were washed three times in ISS and resuspended at $5 \%$ in ISS. RBCs from healthy patients were tested as negative control and identified as normal RBC. Blood from AIHA patients (positive DAT) was collected for testing positive RBC samples, and is referred to as AIHA RBC. Both RBCs samples were prepared as described above. A commercial reagent $\left(\right.$ Diamed $^{\circledR}$ ) was employed as positive control and identified as control cell.

\subsection{Immobilization of $\mathrm{mAbIMUG}$}

The monoclonal antibody against human immunoglobulin (mAbIMUG) was immobilized on the SF layer deposited onto a quartz substrate, which had been treated with a 1:1:5 solution of $\mathrm{NH}_{4} \mathrm{OH}: \mathrm{H}_{2} \mathrm{O}_{2}: \mathrm{H}_{2} \mathrm{O}$ for $10 \mathrm{~min}$ at $70{ }^{\circ} \mathrm{C}$, and then with a 1:1:6 solution of $\mathrm{HCl}: \mathrm{H}_{2} \mathrm{O}_{2}: \mathrm{H}_{2} \mathrm{O}$ for $10 \mathrm{~min}$ at $70{ }^{\circ} \mathrm{C}$. The deposition process was carried out by immersing the substrate in SF and mAbIMUG solutions for $10 \mathrm{~min}$ each, at room temperature. After deposition the film was washed with Milli-Q water to remove poorly adsorbed molecules and dried gently with a nitrogen flow. A step of washing and drying was performed after each deposited layer. The immobilization of mAbIMUG antibody was confirmed by fluorescence spectroscopy using a Fluorolog FL3-22 Jobin-Yvon spectrometer.

\subsection{Electrochemical Measurements}

SF and mAbIMUG were deposited on screen-printed carbon electrodes as described above, but using a Flow-Cell to deposit the materials just onto the working electrode. Flow-Cell and electrodes (model DRP110) were purchased from Dropsens (Oviedo, Spain). Square wave voltammograms were obtained using a Bipotentiostat/Galvanostat 
$\mu$ Stat 400 with DropView software from DropSens (Oviedo, Spain). The measurements were performed using PBS buffer as electrolytic solution, using a scan speed of $0.05 \mathrm{~V} / \mathrm{s}$ within the potential range from -0.6 to $0.6 \mathrm{~V}$. Prior to the measurements, $100 \mu \mathrm{L}$ of red blood cell (RBCs) samples were added onto the films for $5 \mathrm{~min}$, and then washed with PBS buffer.

\subsection{Electrical Measurements}

Impedance spectroscopy measurements were carried out with a Solartron 1260A impedance/gain phase analyzer in a frequency range from 1 to $10^{5} \mathrm{~Hz}$. The measurements were performed using interdigitated electrodes (IDE) made of 50 pairs of gold electrodes $(10 \mu \mathrm{m}$ in width and $200 \mathrm{~nm}$ in thickness) that are $10 \mu \mathrm{m}$ apart from each other coated with mAbIMUG immobilized on the SF layer. The experiments were performed by adding RBCs samples (healthy, sick and control) on the coated interdigitated electrode for $5 \mathrm{~min}$., and then the impedance measurements were taken. In another series of experiments, the measurements were only taken after the electrodes had been washed with a PBS solution after incubation with the RBCs. The objective of this latter series was to verify if irreversible changes occurred with incubation. The dielectric loss and capacitance values were obtained by modeling the impedance response with an equivalent circuit. ${ }^{24}$ Optical images were obtained using a Leica DM 2500M optical microscope coupled to a Leica DFC310 FX camera. The images were collected from interdigitated electrodes after washing with PBS buffer solution.

\section{RESULTS AND DISCUSSION}

\subsection{Immobilization of SF and mAbIMUG}

The silk fibroin (SF) protein produced by Bombyx mori consists basically of 12 repeating Gly-X domains, where $\mathrm{X}$ is Ala, Ser and Tyr in 65\%, 23\% and $9 \%$ of the domains, respectively, being linked by 11 amorphous domains containing tryptophan and charged residues. ${ }^{25}$ Its emission is assigned to the amino acids tyrosine and tryptophan and depends on the crystalline form. In aqueous solution, the emission peaks at $298 \mathrm{~nm}$ as shown in Figure 1(A), while for a deposited film on a quartz substrate the peak appears at $303 \mathrm{~nm}$ in Figure 1(B). This shift is attributed to structural changes from random coils and $\alpha$-helices in solution to $\beta$-sheets in the film. ${ }^{26}$ Also shown in Figure 1 are the emission spectra for mAbIMUG in aqueous solution and immobilized onto the SF film on a quartz substrate, whose peak appears at $326 \mathrm{~nm}$. That similar spectra are obtained both in solution and in the film indicates no conformational change in mAbIMUG upon adsorption on the SF layer. Therefore, SF is confirmed as being a suitable immobilization matrix for antibodies as reported for peptides. ${ }^{16-18,26}$

The growth of layer-by-layer (LbL) films with $\mathrm{SF} / \mathrm{mAbIMUG}$ bilayers on a quartz substrate is depicted in Figure 2, where the emission maximum at $326 \mathrm{~nm}$ increases linearly up to 5 layers $\left(\mathrm{SF}_{3} / \mathrm{mAbIMUG}_{2}\right)$. Adsorption of the sixth mAbIMUG layer caused desorption of the previous SF layer, and the film could no longer grow. This did not represent a problem because electrical and electrochemical immunosensors could be obtained with one or two bilayers, as discussed below.
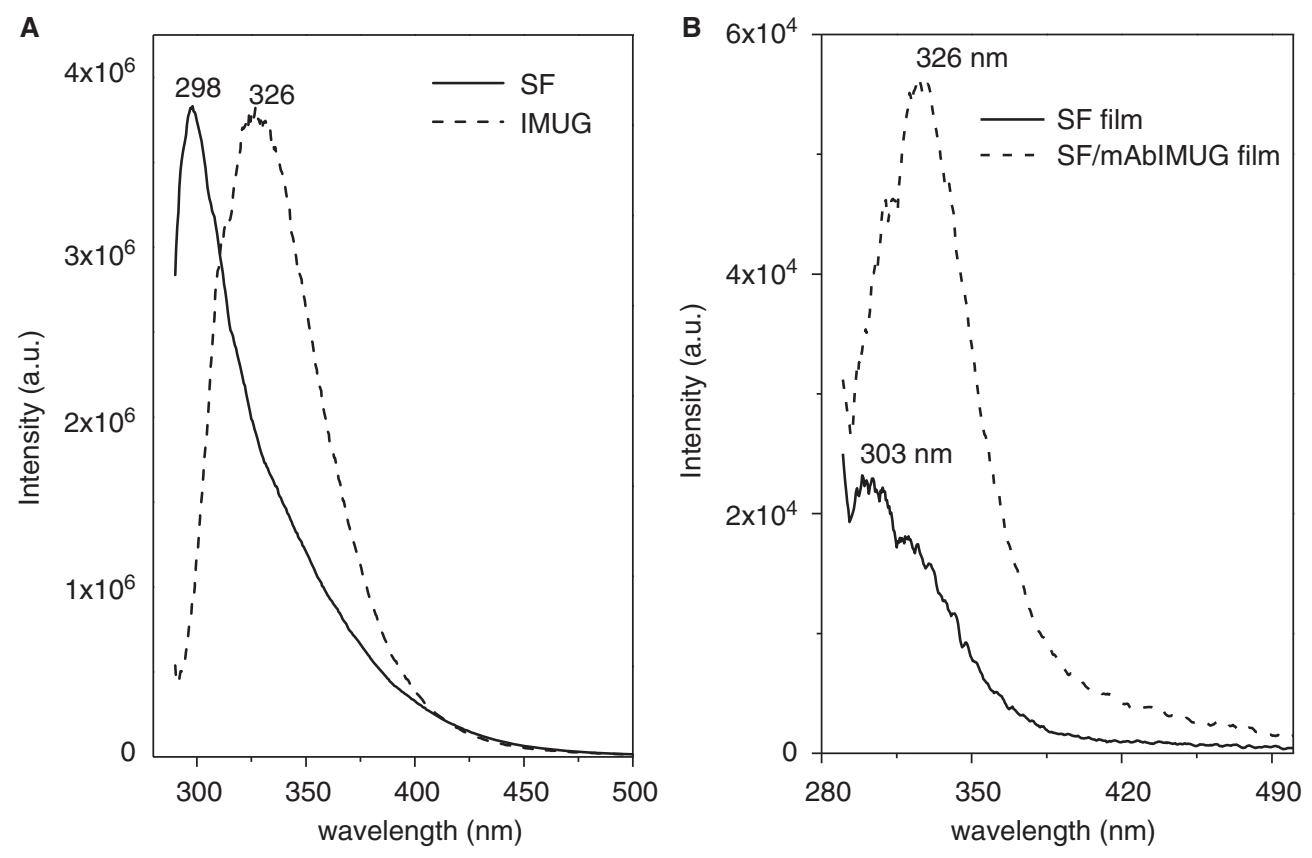

Figure 1. Fluorescence spectra of silk fibroin (solid line) and mAbIMUG antibody (dashed line) in solution (A) and immobilized onto a quartz substrate (B). Excitation at $280 \mathrm{~nm}$. 


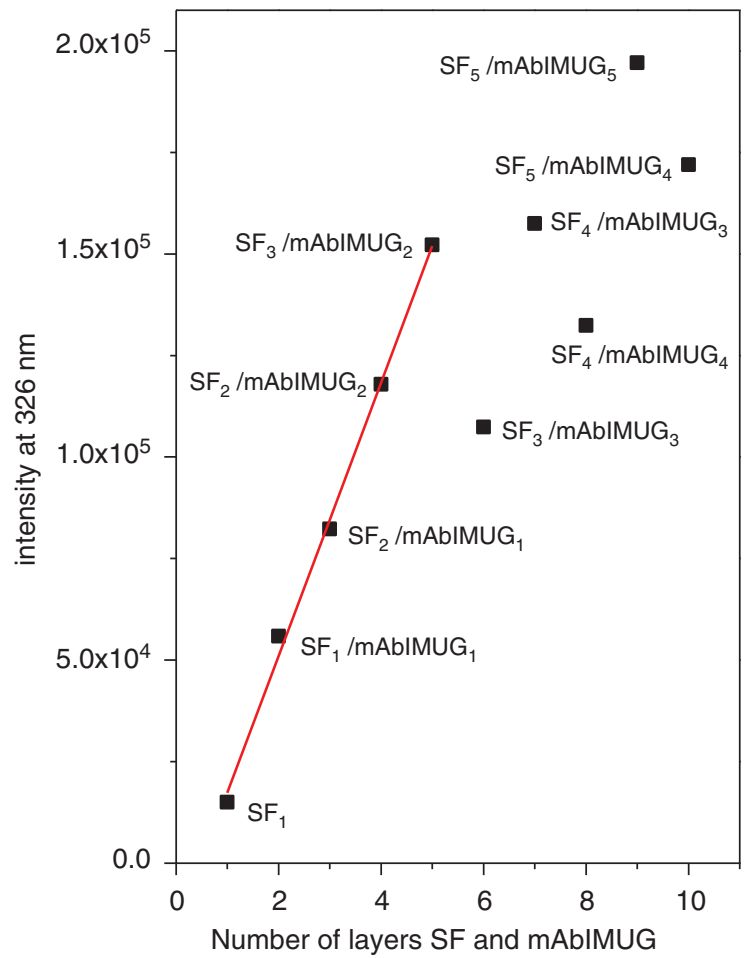

Figure 2. Emission maximum at $326 \mathrm{~nm}$ as a function of the number of SF/mAbIMUG bilayers, where the first layer is SF.

\subsection{Immunosensors Response \\ Immunosensors were prepared with SF/mAbIMUGLbL} films containing one or two bilayers. Apparently, the coverage provided by one bilayer was not sufficient for detection using electrochemistry, and therefore only the 2-bilayer film deposited on a screen printed carbon electrode was used. In these experiments, the electrode was exposed to either normal or sick RBC samples for $5 \mathrm{~min}$, followed by washing procedures with PBS buffer. All measurements were performed after washing the electrodes with PBS buffer. Figure 3(A) shows half-cycles of square wave voltammetry where no reduction or oxidation peaks were observed for electrodes exposed to normal $\mathrm{RBC}$ or PBS buffer. In contrast, a well-defined oxidation peak appeared at $-0.25 \mathrm{~V}$ for the electrode exposed to sick RBCs, which is ascribed to RBC cells attached onto the surface (AIHA RBC). Note that only half-cycles were shown because the process is irreversible. In subsidiary experiments we observed no electrochemical signal when sick RBCs were dispensed onto an uncoated screen printed carbon electrode (bare) or coated only with a SF layer (Fig. 3(B)). This confirms the specificity of the immunosensor, which requires the molecular-recognition interaction between AbmIMUG and sick RBCs.

For the immunosensor based on impedance spectroscopy, optimized performance was obtained with 1-bilayer SF/mAbIMUGLbL film deposited on a gold interdigitated electrode (IDE). The frequency dependence
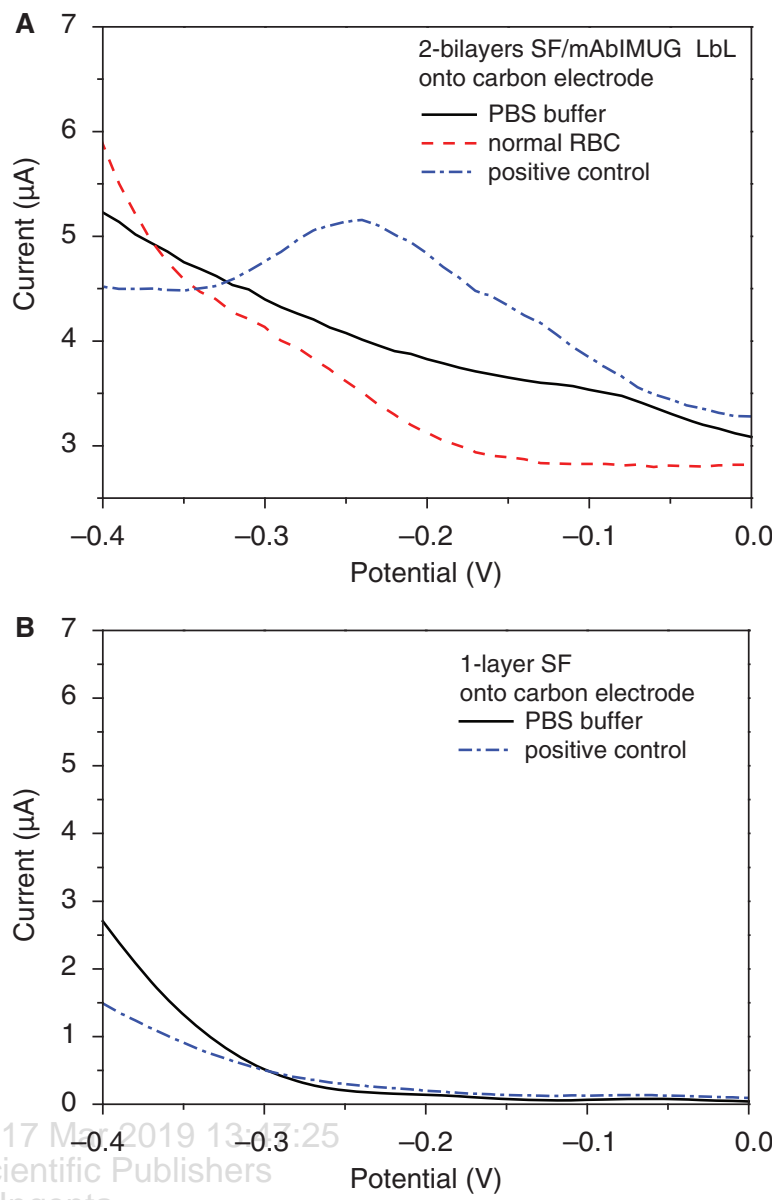

Figure 3. Square wave voltammograms (direct current) for 2-bilayer SF/mAbIMUGLbL films (A) and 1-layer SF (B) on screen printed carbon electrode exposed only to PBS buffer, to normal RBCs and sick RBCs (positive control).

of the dielectric loss in Figure 4 shows a clear shift in the relaxation peak when either sick RBCs or positive control were exposed to the electrode, compared to the PBS buffer and normal RBC samples.

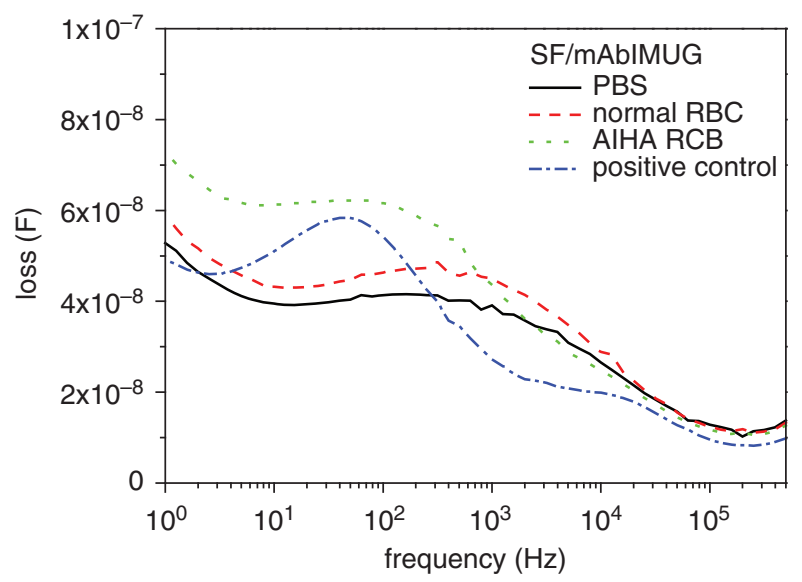

Figure 4. Dielectric loss for one-bilayer SF/mAbIMUGLbL films in the presence of PBS buffer, normal RBCs, AIHA sample and positive control. 

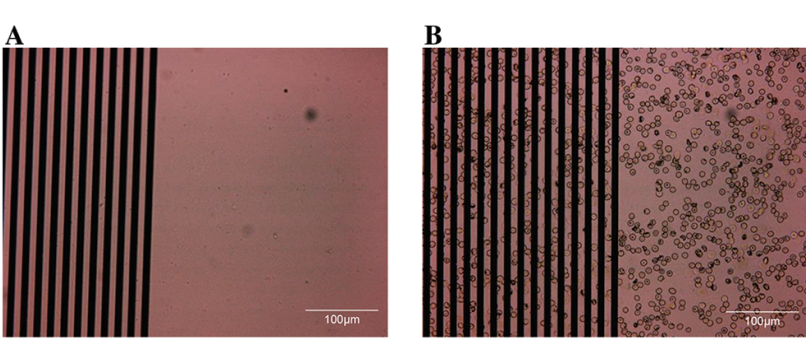

Figure 5. Optical microscopy images of interdigitated electrodes containing one-bilayer SF/mAbIMUG LbL film after exposure to normal RBCs (A) and AIHA-RBCs (B) and washing with PBS buffer.

Both the electrochemical signal and the shift in the relaxation peak may be attributed to adsorption of sick RBC cells on the electrodes, and this is confirmed with the optical microscopy images in Figure 5. Indeed, no cell attachment could be noted on the images of one-bilayer $\mathrm{SF} / \mathrm{mAbIMUG}$ film deposited on the interdigitated electrode after exposure to normal RBC samples. In contrast, AIHA-RBCs were attached so strongly on the electrode surface, as seen in Figure 5(B), that even after washing in PBS buffer, the optical images still pointed to covered electrodes.

\section{CONCLUSIONS}

Silk fibroin (SF) has been confirmedy/as a suitable matrix for immobilization of biomolecules in layer-bylayer (LbL) films. In this study, the monoclonal antibody mAbIMUG immobilized in LbL films, alternated with SF layers, could recognize sick red blood cells (RBCs). This biorecognition was proven with two detection methods, namely square wave voltammetry and impedance spectroscopy. With various control experiments, we could ensure that the electrical and electrochemical response of mAbIMUG-containing LbL films was only altered when adsorption of sick RBCs occurred. The strategy is thus suitable for an effective diagnosis of immune hemolytic anemia (IHA).

Acknowledgments: This work was supported by FAPESP (2013/14262-7), CNPq and Nanobiotec-CAPES network (Brazil). The authors are also grateful to the microfabrication laboratory (LMF/LNNano) (Brazil) for providing interdigitated electrodes (project LMF 12298).

\section{References and Notes}

1. N. R. Rose and I. R. Mackay, The Autoimmune Diseases, 5th edn., Elsevier Academic Press, St. Louis, London, MO (2013).

2. H. Packman, Transfus. Med. Hemother. 5, 317 (2015).

3. H. A. Liebman and I. C. Weitz, Med. Clin. N. Am. 101, 351 (2017).

4. K. Lechner and U. Jager, Blood 116, 1831 (2010).

5. G. F. Bass, E. T. Tuscano, and J. M. Tuscano, Autoimmun. Rev. 13, 560 (2014).

6. E. M. Meulenbroek, D. Wouters, and S. S. Zeerleder, Blood Rev. 29, 369 (2015).

7. W. Barcellini and B. Fattizzo, Expert Opin. Orphan Drugs 6, 273 (2018).

8. A. Pruss, A. Salama, N. Ahrens, A. Hansen, H. Kiesewetter, J. Koscielny, and T. Dörner, Clin. Exp. Med. 3, 55 (2003).

9. R. R. A. Coombs, A. E. Mourant, and R. R. Race, Br. J. Exp. Pathol. 26, 255 (1945).

10. S. Berentsen and G. E. Tjønnfjord, Blood Rev. 26, 107 (2012).

11. Y. Wan, Y. Su, X. Zhu, G. Liu, and C. Fan, Biosens. Bioelectron. 47, 1 (2013).

12. H. Alizadehzeinabad, H. Ghourchian, M. Falahati, M. Fathipour, M. Azizi, and S. M. Boutorabi, Nanotechnol. 29, 265102 (2018).

13. H. Fan, Z. Guo, L. Gao, Y. Zhang, D. Fan, G. Ji, B. Du, and Q. Wei, Biosens. Bioelectron. 64, 51 (2015).

14. C. I. Justino, A. C. Duarte, and T. A. Rocha-Santos, Adv. Clin. Chem. 73, 65 (2016).

15. C. Fang, L. Cao, and Z. Chen, J. Nanosci. Nanotechnol. 17, 5109 (2017).

16. K. Ariga, Y. Yamauchi, G. Rydzek, Q. Ji, Y. Yonamine, K. C.-W. Wu, and J. P. Hill, Chem. Lett. 43, 36 (2014).

17. J. M. Gonçalves, R. L. Lima, M. L. Moraesm, and S. J. L. Ribeiro, Mater. Sci. Eng. C 68, 338 (2016).

18. J. A. Delezuk, A. Pavinatto, M. L. Moraes, F. M. Shimizu, V. C. Rodrigues, S. P. Campana-Filho, S. J. L. Ribeiro, and O. N. Oliveira Jr., Carbohydr. Polym. 155, 146 (2017).

19. J. Chen, G. Cheng, R. Liu, Y. Zheng, M. Huang, Y. Yi, X. Shi, Y. Du, and H. Deng, J. Colloid Interface Sci. 523, 208 (2018).

20. R. Almeida, C. N. Nakamura, M. L. Fontes, E. Deffune, S. L. Felisbino, R. Kaneno, W. J. Fávaro, A. Billis, M. O. Cerri, A. M. Fusco-Almeida, M. J. M. Giannini, and A. Moroz, mAbs 10, 46 (2018).

21. D. N. Rockwood, R. C. Preda, T. Yücel, X. Wang, M. L. Lovett, and D. L. Kaplan, Nat. Protoc. 6, 1612 (2011).

22. G. KöHler and C. Milstein, Nature 256, 495 (1975).

23. E. Harlow and D. Lane, Antibodies a Laboratory Manual, Cold Spring Harbor Laboratory, Cold Spring Harbor, N.Y. (1988).

24. D. M. Taylor and A. G. Macdonald, J. Phys. D: App. Phys. 20, 1277 (1987).

25. F. G. Omenetto and D. L. Kaplan, Science 329, 528 (2010).

26. M. L. Moraes, L. R. Lima, R. R. Silva, M. Cavicchioli, and S. J. L. Ribeiro, Langmuir 29, 3829 (2013).

Received: 23 April 2018. Accepted: 25 June 2018. 